\title{
ON FINITE CAPACITY QUEUEING SYSTEMS WITH A GENERAL VACATION POLICY
}

\author{
JACQUELINE LORIS-TEGHEM \\ University of Mons-Hainaut \\ Department of Applied Mathematics \\ Place Warocqué, 17 B-7000, Monz Belgium \\ E-mail: Jacqueline.Loris@umh.ac.be
}

(Received December, 1999; Revised May, 2000)

\begin{abstract}
We consider a Poisson arrival queueing system with finite capacity and a general vacation policy as described in Loris-Teghem [Queueing Systems 3 (1988), 41-52]. From our previous results regarding the stationary queue length distributions immediately after a departure and at an arbitrary epoch, we derive a relation between both distributions which extends a result given in Frey and Takahashi [Operations Research Letters 21 (1997), 95-100] for the particular case of an exhaustive service multiple vacation policy.
\end{abstract}

Key words: Finite Capacity Queue, General Vacation Policy, Queue Length.

AMS subject classifications: $60 \mathrm{~K} 25$.

\section{Introduction}

For the M/GI/1 queueing system with finite capacity and exhaustive service multiple vacation policy studied previously by Courtois [1] and Lee [3], Frey and Takahashi [2] analyze the stationary queue length distribution at an arbitrary epoch by expressing it in terms of the stationary queue length distribution at service completion epochs, for which they provide a system of recurrence equations.

According to them, they would be first to consider the departure epoch imbedded Markov chain for vacation models with finite capacity. We would like to emphasize that in Loris-Teghem [5], dealing with a finite capacity queueing system with a general vacation policy, we derived the stationary queue length distribution immediately after a departure and at an arbitrary epoch, by relating each of both distributions to the corresponding distribution in the model without vacations.

In the present note, we use our previous results to express the stationary queue length distribution at an arbitrary epoch in terms of the stationary queue length distribution immediately after a departure, thus extending to the general vacation policy 
a result obtained in Frey and Takahashi [2] for the exhaustive service multiple vacation policy.

\section{The Model}

We consider a queueing system with a Poisson arrival process (with rate $\lambda$ ) and a finite capacity $(L)$. We assume that at time $t=0$, a departure occurs with $\varepsilon$ customers left in the system (where $\varepsilon$ is a fixed non-negative integer, with $\varepsilon<L$ ), and an inactive phase then begins for the server, who will become active again at time $\tau_{1}$. The active phase initiated at $\tau_{1}$ will end the first time a departure occurs with $\varepsilon$ customers left in the system. Thus the server is alternatively in the inactive and active states.

Let $0=t_{0}<t_{1}<\ldots<t_{n}<\ldots\left(0<\tau_{1}<\ldots<\tau_{n}<\ldots\right)$ be the epochs at which the server "enters" the inactive (active) state and $x_{n}, n \geq 1$, the number of customers present in the system at time $\tau_{n}+0$. Let $\nu_{n}=\tau_{n}-t_{n-1}(n \geq 1)$. We make the following further assumptions:

- the epochs $t_{n}$ are the times at which the number of customers in the system decreases to $\varepsilon$. (For $\varepsilon>0$, service is non-exhaustive);

- $\quad$ the random variables $\nu_{n}, n \geq 1$, are i.i.d. with $\varepsilon+1 \leq x_{n} \leq L$ a.s. and with finite expectation;

- the service times are i.i.d. random variables - with finite expectation $E(S)$ independent of the arrival process and of the sequence $\left\{\left(\nu_{n}, x_{n}\right), n \geq 1\right\}$;

- $\quad$ service is non-preemptive and customers are served in an order independent of their service times.

\section{Queue Length Distributions}

We first consider the Markov chain $\left\{l_{n}, n \geq 1\right\}$, where $l_{n}$ denotes the number of customers in the system immediately after the $n$th departure. As proved in LorisTeghem [5], the stationary distribution of $\left\{l_{n}, n \geq 1\right\}$ - denoted by $\left\{\pi_{\nu, \varepsilon}^{L}(j), j=\right.$ $\varepsilon, \ldots, L-1\}$ - is related to the corresponding distribution in the $\mathrm{M} / \mathrm{GI} / 1 / \mathrm{L}$ model without vacations - denoted by $\left\{\pi^{L}(j), j=0, \ldots, L-1\right\}$ - according to the following formula:

$$
\pi_{\nu, \varepsilon}^{L}(j)=\left(a_{\nu, \varepsilon}^{L}\right)^{-1} \alpha_{L-\varepsilon} \sum_{i=\varepsilon}^{j} \operatorname{Pr}[x>i] \pi^{L-\varepsilon}(j-i) \quad(j=\varepsilon, \ldots, L-1)
$$

where:

- $\quad x$ is distributed as the $x_{n}, n \geq 1$;

- $\quad a_{\nu, \varepsilon}^{L}$ is the expected duration of an active phase;

- $\alpha_{m}$ is the expected duration of the busy period in the $\mathrm{M} / \mathrm{GI} / 1 / \mathrm{m}$ model without vacations.

We now consider the stationary distribution of the queue length at an arbitrary epoch - denoted by $\left\{p_{\nu, \varepsilon}^{L}(j), j=\varepsilon, \ldots, L\right\}$. As proved in Loris-Teghem [5], it is related to the corresponding distribution in the $\mathrm{M} / \mathrm{GI} / 1 / \mathrm{L}$ model without vacations denoted by $\left\{p^{L}(j), j=0, \ldots, L\right\}$ - according to the following formula:

$$
p_{\nu, \varepsilon}^{L}(j)=\left(\lambda c_{\nu, \varepsilon}^{L}\right)^{-1}\left(1+\lambda \alpha_{L-\varepsilon}\right) \sum_{i=\varepsilon}^{j} \operatorname{Pr}[x>i] p^{L-\varepsilon}(j-i) \quad(j=\varepsilon, \ldots, L-1)
$$


and another formula for $j=L$, which will not be used here, where $c_{\nu, \varepsilon}^{L}$ is the expected duration of a cycle.

We will now express the distribution $\left\{p_{\nu, \varepsilon}^{L}(j), j=\varepsilon, \ldots, L\right\}$ in terms of the distribution $\left\{\pi_{\nu, \varepsilon}^{L}(j), j=\varepsilon, \ldots, L-1\right\}$, using relations (1) and (2), and the following relations for the $\mathrm{M} / \mathrm{GI} / 1 / L-\varepsilon$ system without vacations (see Loris-Teghem [4]):

$$
\begin{gathered}
\pi^{L-\varepsilon}(r)=\frac{p^{L-\varepsilon}(r)}{1-p^{L-\varepsilon}(L-\varepsilon)} \quad(r=0, \ldots, L-\varepsilon-1) \\
\frac{\alpha_{L-\varepsilon}}{1-p^{L-\varepsilon}(L-\varepsilon)}=E(S)\left(1+\lambda \alpha_{L-\varepsilon}\right) .
\end{gathered}
$$

Using (3) and (4) in (1), we obtain

$$
\pi_{\nu, \varepsilon}^{L}(j)=\left(a_{\nu, \varepsilon}^{L}\right)^{-1} E(S)\left(1+\lambda \alpha_{L-\varepsilon}\right) \sum_{i=\varepsilon}^{j} \operatorname{Pr}[x>i] p^{L-\varepsilon}(j-i)
$$

which, together with (2), yields

$$
p_{\nu, \varepsilon}^{L}(j)=\pi_{\nu, \varepsilon}^{L}(j)(\lambda E(S))^{-1} a_{\nu, \varepsilon}^{L}\left(c_{\nu, \varepsilon}^{L}\right)^{-1}
$$

which can also be written as

$$
p_{\nu, \varepsilon}^{L}(j)=\pi_{\nu, \varepsilon}^{L}(j)(\lambda E(S))^{-1} \frac{1}{1+d_{\nu, \varepsilon}^{L}\left(a_{\nu, \varepsilon}^{L}\right)^{-1}}
$$

with $d_{\nu, \varepsilon}^{L}$ denoting the expected duration of an inactive phase.

Using (1) for $j=\varepsilon$ and taking into account that $E(S)=\alpha_{m} \pi^{m}(0)$ for $m \geq 1$ (see [4]), we obtain

$$
\left(a_{\nu, \varepsilon}^{L}\right)^{-1}=\pi_{\nu, \varepsilon}^{L}(\varepsilon)(E(S))^{-1}
$$

so that

$$
\begin{gathered}
p_{\nu, \varepsilon}^{L}(j)=\pi_{\nu, \varepsilon}^{L}(j) \frac{\lambda^{-1}}{E(S)+d_{\nu, \varepsilon}^{L} \pi_{\nu, \varepsilon}^{L}(\varepsilon)}(j=\varepsilon, \ldots, L-1) \\
p_{\nu, \varepsilon}^{L}(L)=1-\frac{\lambda^{-1}}{E(S)+d_{\nu, \varepsilon}^{L} \pi_{\nu, \varepsilon}^{L}(\varepsilon)}
\end{gathered}
$$

These relations extend to the general vacation policy considered here, relations (13) and (14) obtained in Frey and Takahashi [2] for the exhaustive service multiple vacation policy. In this particular case, $\varepsilon=0$ and $\nu_{n}$ is distributed as $\sum_{i=1}^{q}{ }_{n} u_{n, i}$, where the random variables $u_{n, i}(n \geq 1, i \geq 1)$ are i.i.d. and $q_{n}$ is the smallest integer verifying the condition that the system is non-empty at time $\left(t_{n-1}+\sum_{i=1}^{q}{ }_{n, i} u_{n,}\right.$. Thus

$$
d_{\nu, \varepsilon}^{L}=d_{\nu, 0}^{L}=E(U)\left(1-b_{0}\right)^{-1}
$$

where $E(U)=E\left(u_{n, i}\right)$ and $b_{0}$ is the probability that no arrival occurs in a time 
interval with a random length distributed as the $u_{n, i}$.

\section{References}

[1] Courtois, P.J., The M/G/1 finite capacity queue with delays, IEEE Trans. Commun. COM-28 (1980), 165-172.

[2] Frey, A. and Takahashi, Y., A note on an M/GI/1/N queue with vacation time and exhaustive service discipline, Oper. Res. Letters 21 (1997), 95-100.

[3] Lee, T., M/G/1/N queue with vacation time and exhaustive service discipline, Oper. Res. Letters 32 (1984), 774-784.

[4] Loris-Teghem, J., Imbedded and non-imbedded stationary distributions in a finite capacity queueing system with removable server, Cah. Centre Et. Rech. Op. 26 (1984), 87-94.

[5] Loris-Teghem, J., Vacation policies in an M/G/1 type queueing system with finite capacity, Queueing Systems 3 (1998), 41-52. 


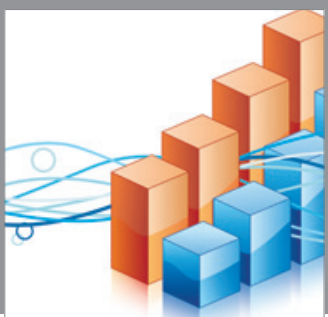

Advances in

Operations Research

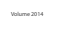

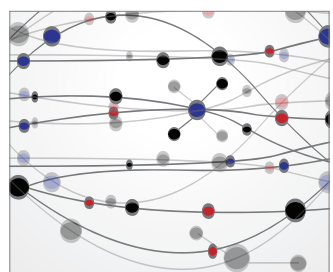

\section{The Scientific} World Journal
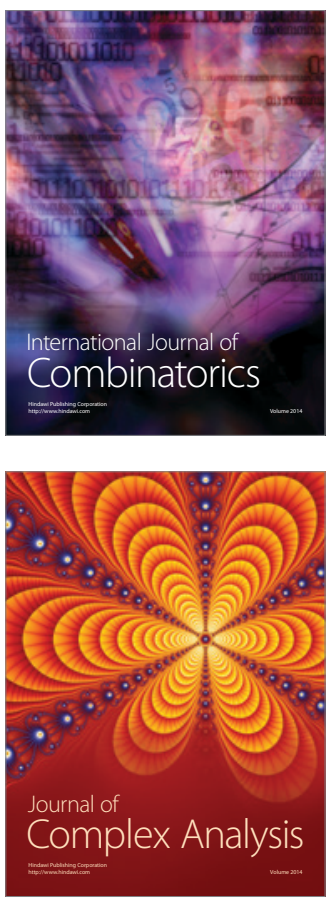

International Journal of

Mathematics and

Mathematical

Sciences
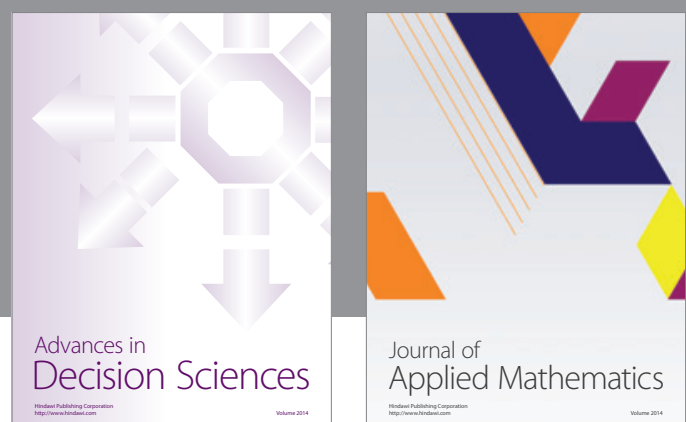

Journal of

Applied Mathematics
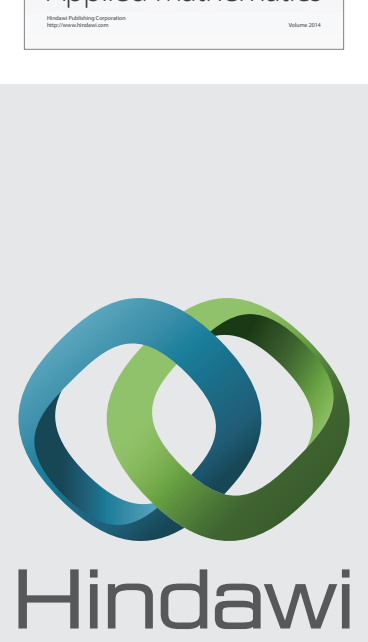

Submit your manuscripts at http://www.hindawi.com
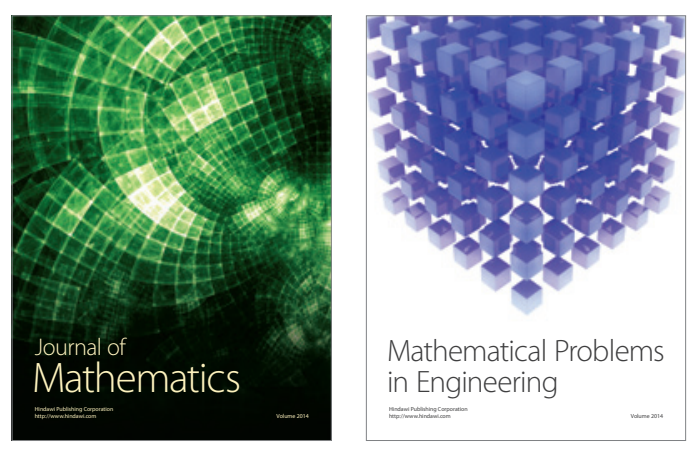

Mathematical Problems in Engineering
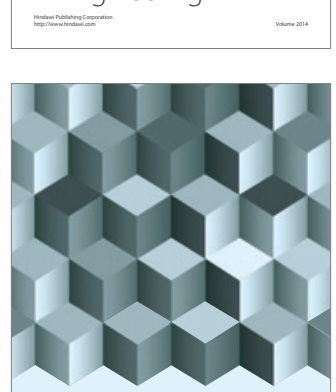

Journal of

Function Spaces
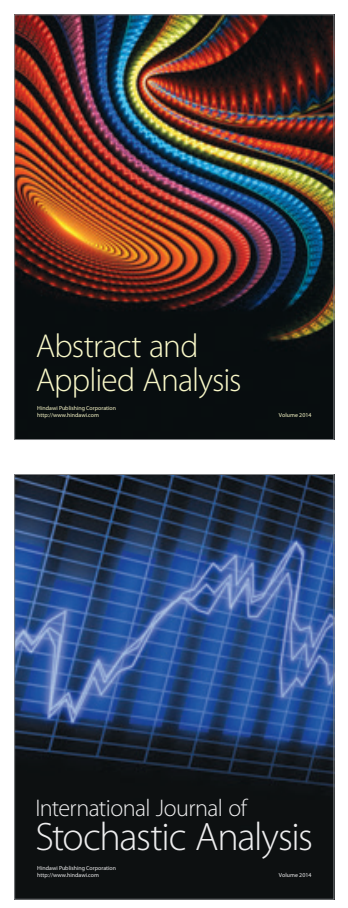

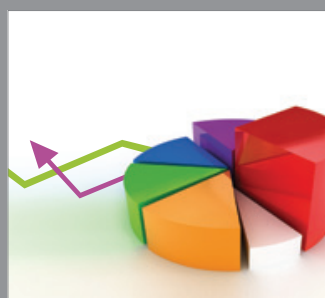

ournal of

Probability and Statistics

Promensencen
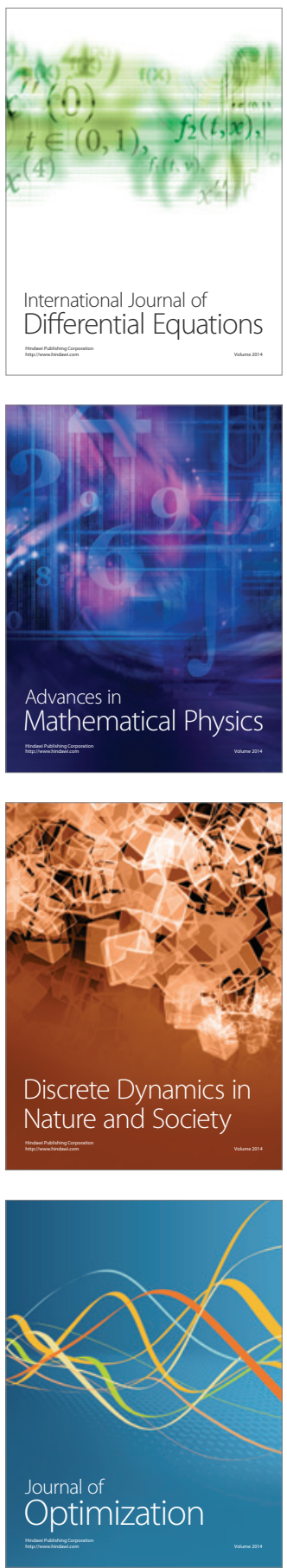